\title{
Transient Stratified Flow in a Differentially-Heated Rectangle with Highly-Conducting Horizontal Walls
}

\author{
Jun Sang Park \\ HallaUniversity \\ 28 Halladaegil, Wonju, Gangwondo, 26404, Korea \\ jspark@halla.ac.kr
}

\section{Extended Abstract}

Buoyant convection of a viscous incompressible fluid in an enclosed rectangle is characterized by three principal non-dimensional parameters, i.e., the Rayleigh number, the Prandtl number, and the container aspect ratio. The majority of previous investigations have dealt with steady flows at large Rayleigh number, [e.g., Batchelor [1]; Elder [2]; Gill[3]], and works on transient situations are not relatively numerous, [see Hyun [4]].

The pioneering efforts by Sakurai \& Matsuda [5] and Jischke \& Doty [6] addressed the linearized heat-up problem, in which the temperature at the vertical wall changes abruptly to another profile. It was demonstrated that adjustment of the bulk of interior with a pre-existing stable stratification is substantially accomplished over the heat-up timescale, which is an order-of-magnitude smaller than the diffusive timescale.

In related developments, Patterson \& Imberger [7] performed an elaborate scale analysis of evolution of an initially isothermal fluid subject to a sudden differential heating of the vertical walls. In these problem formulations, the horizontal walls were taken to be insulating.

The above theoretical expositions assumed that the horizontal walls are passive in nature, and, therefore, no explicit delineation was made of the flow near the horizontal walls [e.g., Hyun [4]]. The crux of argument here is that the usual assumption of passive horizontal walls should be viewed with caution. It will be shown in this paper that, under certain circumstances, the horizontal walls take a more active role in the determination of transient interior flows even if the problem is linear.

An overview is given to illuminate the dynamical role of horizontal walls. For this purpose, two paradigmatic layouts of fluid adjustment process, in response to an abrupt increase of temperature at the vertical wall, are considered. In type A, the change in the vertical wall temperature is linear along the vertical coordinate $Z$, i.e., $T_{*}^{*} \rightarrow T_{*}^{*}+\varepsilon \cdot z \cdot \Delta T^{*}$; and in type B, the change is uniform, i.e., $T_{*}^{*} \rightarrow T_{*}^{*}+\varepsilon \cdot \Delta T^{*}$. In the above, $T^{*}$ denotes the pre-existing temperature profile at the initial equilibrium state of the stratified fluid, and $\varepsilon(\ll 1)$ indicates the Rossby number which measures the strength of perturbations.

Type A was first studied by Sakurai \& Matsuda [5], and analogy exists between this problem and the classical spinup in rotating fluids [e.g., Greenspan \& Howard [8]]. In the transient stage, motions in the interior far away from the wall are induced by the divergent Rayleigh boundary layer at the wall. The interior flows are determined exclusively by the conditions at the vertical wall, and the conditions at the horizontal walls exert no effect on the interior motions. Since the vertical fluid transport in the divergent Rayleigh layer varies with height, the horizontal fluid motions in the interior are determined such that the vertical mass continuity at every height is satisfied. In other words, pumping by the Rayleigh layer directly controls the interior fluid motions. Consequently, the presence or absence of horizontal walls does not affect the global flow patterns in the interior. This is manifested in the passiveness of horizontal walls.

In type B, a non-divergent buoyancy layer appears on the vertical wall, which does not pump the interior fluid directly [e.g., Park \& Hyun [9]]. Therefore, the character of interior flow in a finite cavity is qualitatively different from that in an infinite container. These observations suggest that the horizontal walls actively participate in the determination of interior flows for type B. However, the subtle nature of the role of horizontal walls should be underscored; the activeness of the horizontal walls comes into the picture to fulfill the kinematic conditions rather than to induce the dynamicallysignificant buoyancy. In summary, in type B-problems, a more comprehensive analysis of horizontal boundary layer is in order, which can be characterized as the horizontal wall being kinematical active but dynamical passive. This task has not 
been properly elucidated in the literature, and this forms the principal effort of the present paper.

The thrust of the entire paper is placed on analytical descriptions of transient features over the timescale of formation

of buoyancy layer $O\left(N^{-1}\right)$ and the heat-up timescale $O\left(R_{a}^{1 / 4} N^{-1}\right)$, where $R_{a}$ denotes the system Rayleigh number and $N$ the Brunt-vaisala frequency. It is important to note that, in the present problem formulation, the buoyancy layer is the principal driver of interior motions. Therefore, in this paper, a theoretical analysis will be made of transient motions over the timescale of formation of buoyancy layer, $O\left(N^{-1}\right)$. Based on the theoretical results of two timescale analysis, appropriate physical rationalizations are given and also descriptions of the complete picture of the heat-up evolution are given(Xu \& Saha[10], Liu et al.[11]) .

\section{Acknowledgement}

This research was supported in part by National Research Foundation of Korea (NRF-2015R1D1A1A02062408)

\section{References}

[1] G. K. Batchelor, "Heat transfer by free convection across a closed cavity between vertical boundaries at different temperature," Quart. Applied Math., vol.12, pp. 209-233, 1954.

[2] J. W. Elder, "Laminar free convection in a vertical slot," J. Fluid Mech., vol. 23, pp. 77-98, 1965.

[3] A.E. Gill, "The boundary layer regime for convection in a rectangular cavity," J. Fluid Mech., vol. 26, pp. 515-536, 1966.

[4] J. M. Hyun, "Unsteady buoyant convection in an enclosure," Advances in heat transfer, vol. 24, pp. 277-319, 1994.

[5] T. Sakurai, T. Matsuda, "A temperature adjustment process in a Boussinesq fluid via a bouncy-induced meridional circulation.," J. Fluid Mech., vol. 54, pp. 417-421, 1972.

[6] M. C. Jischke and R.T. Doty, "Linearized buoyant motion in a closed container," J. Fluid Mech., vol. 71, pp. 729-754, 1975.

[7] J. Patterson, "J. Imberger, Unsteady natural convection in a rectangular cavity," J. Fluid Mech., vol. 100, pp. 65-86, 1980.

[8] H. P. Greenspan and L.N. Howard, "On a time dependent motion of a rotating fluid," J. Fluid Mech., vol. 17, pp. 385-404, 1963.

[9] J. S. Park and J. M. Hyun, "Transient process of a confined stratified fluid induced simultaneously by sidewall thermal loading and vertical through-flow," J. Fluid Mech., vol. 451, pp. 295-317, 2002.

[10] F. Xu and S.C. Saha, "Transition to an unsteady flow induced by a fin on the sidewall of a differentially heated airfilled square cavity and heat transfer," Int. J. Heat \& Mass Transfer, vol. 71, pp. 236-244, 2014.

[11] Y. Liu, C. Lei and J. C. Patterson, "Natural convection in a differentially heated cavity with two horizontal adiabatic fins on the sidewalls," Int. J. Heat \& Mass Transfer, vol. 71, pp. 23-36, 2014. 